\title{
JOVIAN CAPTURE OF A SPACECRAFT WITH A SELF-BALANCED ELECTRODYNAMIC BARE TETHER
}

\author{
M. Sanjurjo-Rivo ${ }^{*}$, D.J. Scheeres ${ }^{\dagger}$ and J. Peláez ${ }^{\ddagger}$
}

\begin{abstract}
The exploration of Jupiter and the Jovian system constitutes an important challenge of planetary exploration. Among the technological obstacles to deal with, the scarcity of power is one of the most limiting factors. In recent works, electrodynamic tethers have been proposed as an efficient solution to provide power in a permanent manner. Furthermore, they are pointed out as also suitable propulsive devices to perform orbit manoeuvres in the Jovian world.

In this paper, the Jupiter capture of a spacecraft using a self balanced electrodynamic tether as the propulsion system is analyzed in detail.
\end{abstract}

\section{INTRODUCTION}

Missions to the outer planets have to face two important limitations which constrain their operability and their scientific payload. On the one hand, the necessary propellant mass for the capture and, on the other hand, the scarcity of power generation. Both of these have had an important impact on the design of previous missions to Jupiter. The search for solutions to avoid these handicaps led to the proposal of the Jupiter Icy Moons Orbiter mission which enhanced as compared to the performance of former missions. JIMO would have used a small nuclear reactor to overcome the weak electrical power generation and to power ion electric thrusters, improving mission manoeuvering. The cancelation of this mission means the problem is still open and fosters the proposal of alternative new concepts.

In this scenario, the consideration of electrodynamic tethers appear to be a suitable solution since they can target both limitations simultaneously. Firstly, in Peláez and Scheeres ${ }^{1,2}$, a new strategy to provide power in a permanent manner using electrodynamic tethers is fully described and analyzed for a jovian orbit. Furthermore, the utilization of electrodynamic tethers to perform the capture manoeuvre has been studied in several articles. Gallagher et al. ${ }^{3}$ presents a numerical computation of spacecraft trajectories assuming a given attitude for the tether and an "ad hoc" model of the current collection. Besides, they consider retrograde captures and provide a study of the possible thermal side effects on the cable. In turn, Sanmartin and Lorenzini ${ }^{4}$ give a fully analytical estimation of an electrodynamic tether's performance and the main parameters involved in a prograde capture with an accurate model of the current collection as well as some simplifications concerning the geometry of the trajectory and the attitude of the tether. In this work the concept of the drag sphere was presented, inside of which the tether produces drag while outside of which it can generate thrust. In van Dijk et al. ${ }^{5}$ a complete study of the electrodynamic force capabilities of electrodynamic

*Ph.D. Candidate, E.T.S.I. Aeronáuticos, Pza. Cardenal Cisneros 3, E-28040 Madrid, Spain. E-mail address: m.sanjurjo@upm.es

${ }^{\dagger}$ Professor, Colorado Center of Astrodynamics Research, Department of Aerospace Engineering Sciences, The University of Colorado

${ }^{\ddagger}$ Associate Professor, E.T.S.I. Aeronáuticos, Pza. Cardenal Cisneros 3, E-28040 Madrid, Spain. E-mail address: j.pelaez@upm.es. AIAA Member 
tethers in the jovian environment is developed. The attitude is considered here in detail, though the results are applied only to circular orbits. Finally, Sanmartin et al. ${ }^{6}$ is an essential report gathering almost all the important concepts regarding the capture manoeuvre at Jupiter. Nevertheless, it is focused only on rapidly rotating tethers, and therefore obviates the attitude dynamics.

In this paper, the feasibility of using electrodynamic tethers to overwhelm the constraint imposed by the capture manoeuver is analyzed from a broader point of view. In order to achieve that goal, a comprehensive model is derived, gathering the relevance of the attitude dynamics in the jovian capture as well as keeping the accuracy of the environmental models. Some formerly proposed strategies are reviewed in the light of this model and a new capture method is studied based on the SBET (Self Balanced Electrodynamic Tether) concept.

The main characteristic of SBETs is that they avoid the instabilities associated with the attitude dynamics in inclined orbits. In the near Earth environment, SBETs have proven its capability to stabilize electrodynamic tethers, combining high tether intensities with no perturbation torques, and, therefore, with a stable attitude state. This property allows the tether to work aligned with the local vertical which represents an important benefit in terms of current collection efficiency in addition to the desired attitude stability. The consideration of the former criterion lead to the utilization of tethers without insulation, called bare tethers since this solution solves the problem of anodic contact in highly rarified plasma.

The next section is devoted to describing the manoeuvre and the tethered system, comparing the models used with those applied in previous studies. The following sections are aimed to define in detail the complete dynamical model which is used in the analysis of the jovian capture. According to the attitude strategy chosen to perform the capture manoeuvre, the study will be split into rotating and non rotating tethers. For the particular case of rapidly rotating tethers, a specific formulation is proposed. Then, the control options of non rotating tethers are explored in detail, arriving to the establishment of a control strategy. Lastly, a thermal analysis of the cable has been performed. The high currents the tether would provide produce the heating of the cable due mainly to ohmic effects. This element can be a limiting factor of the allowable current which can be borne. Considering this fact, the operational limits for both rotating and non rotating tethers are established.

\section{DESCRIPTION OF THE TETHERED MANOEUVRE}

The electrodynamic tether generates drag only in a fraction of an arc of the whole trajectory. Due to this, the tethered system will follow a hyperbolic trajectory during the first part of the manoeuvre, within the validity of the patched conic approximation. This initial hyperbolic orbit can be defined completely by means of the arrival velocity, $v_{\infty}$, the radius of the periapsis, $r_{p}$, and the orbital plane *. All the foregoing studies, as well as the present, have considered an equatorial plane for the capture manoeuvre since it represents the most effective solution in terms of the electrodynamic work which can be provided. Additionally, the initial relative velocities which have been considered vary from $5.64 \mathrm{~km} / \mathrm{s}$ of the minimum-energy transfer from the Earth (ignoring the inclination of Jupiter's orbit with respect to the ecliptic) in Sanmartin et al. ${ }^{6}$ to $6 \mathrm{~km} / \mathrm{s}$ in Sanmartin and Lorenzini ${ }^{4}$ and to $6.854 \mathrm{~km} / \mathrm{s}$ of a previous concept space mission in Gallagher et al. ${ }^{3}$ In this article we will consider arrival velocities of 1.0 and 1.2 times the velocity of the Hohmann transfer.

On the other hand, the considered radius of periapsis varies from $1.01 R_{J}$ (from a proposed mission Radio Science Observer) in Gallagher et al. ${ }^{3}$ to $1.5 R_{J}$ in Sanmartin and Lorenzini. ${ }^{4}$ In

\footnotetext{
${ }^{*}$ By definition of the jovian reference frame, $\omega+\Omega=0$
} 
retrograde capture orbits, there are no constraints on this parameter, but in prograde orbit it is necessary that the perijove is inside the drag sphere (generally speaking, since for hyperbolic orbits the stationary radius is not defined). From the results of these articles, it can be deduced that the radius of periapsis is a decisive parameter in the design of Jovian capture trajectories. In the current work we will explore the results obtained for the radius of periapsis between $1.0 R_{4}$ and $2.0 R_{4}$.

Regarding the computation of electrodynamic forces, it is necessary to establish a model for current collection. The interaction among a conductive tether, the ionosphere and magnetic field of a planet like Jupiter can lead to the generation of an intensity inside the cable. In a frame orbiting with the tether, there exists an electric field given by:

$$
\overrightarrow{\boldsymbol{E}}_{m}=\left(\overrightarrow{\boldsymbol{v}}_{S C}-\overrightarrow{\boldsymbol{v}}_{p l}\right) \times \overrightarrow{\boldsymbol{B}}
$$

where $\overrightarrow{\boldsymbol{v}}_{S C}$ and $\overrightarrow{\boldsymbol{v}}_{p l}$ are the inertial velocities of the spacecraft and the plasma, respectively. This field can drive a current inside the tether. The study of the mechanisms which determine the tether current collection is out of the scope of this analysis. Still, it should be clarified that the intensity through the cable will be different as a function of the anodic contactors used. There are, basically, two procedures to collect electrons, a balloon at the anodic end of the tether (then the collection follows the Parkers-Murphy current), or a bare tether ${ }^{4,6,5}$ (then the collection takes place in the orbital-motion-limited (OML) regime, as long as some conditions regarding the transversal tether dimensions are fulfilled)* ${ }^{*}$ The latter has been chosen as the preferable option, as it has been discussed before.

In the OML regime, the evolution of the current intensity along the tether is governed by the following equation ${ }^{7}$ :

$$
I_{O M L}=2 R L_{t} n_{\infty} e \sqrt{\frac{2 e \Phi_{p}}{m_{e}}}
$$

where $R$ is the tether radius, $n_{\infty}$ is the electron plasma density, $e$ is electron charge, $m_{e}$ is the electron mass and $\Phi_{p}$ is the tether-plasma bias. Therefore, current collection depends on the differential potential between the cable and the surrounding plasma, and hence, it is necessary to compute its evolution considering the motional electric field along the cable, $E_{m}$, and the ohmic losses due to the intensity. The statement of the full set of differential equations which models the current collection is presented in Sanmartin and Ahedo ${ }^{8}$ and Martinez-Sanchez and Sanmartin ${ }^{9}$. The statement of this problem is completed with boundary conditions at both ends where an hypothetical load for power generation and the voltage drop at the cathodic end appear. As a result, a system of differential equations with boundary conditions must be solved to obtain the current and bias profile along the cable.

Regarding the environment of Jupiter, the magnetic field is generally considered as a no-tilt dipole ${ }^{6,5}$ whose characteristics are described in Divine and Garret ${ }^{10}$. Nevertheless, in Gallagher et al. $^{3}$, a more complex model ${ }^{11}$ is followed. On the other hand, to determine the charge population the work of Divine and Garret ${ }^{10}$ is generally used. Still in Gallagher et al. ${ }^{3}$ a simplified version of a more extended model ${ }^{12}$ has been considered. For a deep discussion about the jovian environmental conditions related to the operation of electrodynamic tethers see Sanmartin et al. ${ }^{6}$, chapter 3.

\section{Tether Model}

The Dumbbell model will be used to describe the tethered system. This model allows to provide a first approximation of the system behavior. The tether is supposed to be a conductive rod with

\footnotetext{
*In Gallagher et al. ${ }^{3}$, a combination of both are considered
} 
mass $m_{t}$ and length $L_{t}$, connecting two end masses $m_{1}$ (the lower mass)and $m_{2}$ (the upper mass). Instead of $m_{1}, m_{2}$ and $m_{t}$ the parameters $m$, the total mass, $\chi$ and $\Gamma$ has been used to describe the configuration of mass, being their definitions:

$$
\begin{aligned}
m & =m_{1}+m_{2}+m_{t} & m_{t} & =m \Gamma \\
m_{1} & =m\left(\cos ^{2}(\chi)-\frac{\Gamma}{2}\right) & m_{2} & =m\left(\sin ^{2}(\chi)-\frac{\Gamma}{2}\right)
\end{aligned}
$$

The maximum and minimum values of $\chi$ are reached when $m_{1}=0$ and $m_{2}=0$, respectively. Then $\chi$ is defined in the interval $\left[\chi_{\min }, \chi_{\max }\right]$ whose values are given by:

$$
\chi_{\min }=\arcsin \left(\frac{\Gamma}{2}\right) \quad \chi_{\max }=\arccos \left(\frac{\Gamma}{2}\right)
$$

In turn, the value of the moments of inertia respect to the direction of the cable is assumed to be zero. For any other perpendicular direction the value of the moment of inertia is $I_{s}$. Expressed in terms of the parameters of the geometry of mass, $I_{s}$ is equal to:

$$
I_{s}=\frac{1}{12} m_{t} L_{t}^{2}\left(3 \sin ^{2}(2 \chi)-2 \Gamma\right)
$$

The more restrictive assumption of the Dumbbell model for the case of study is the hypothesis that the tether remains straight, since important lateral forces are acting during the capture trajectory.

\section{MOTION OF THE CENTER OF MASS}

In this section a description of the dynamics of the center of mass is derived in terms of the osculating orbital elements of the trajectory. This characterization allows us to provide further insight on the manoeuvre as well as develop an easy numerical implementation.

Before exposing the detailed derivation of the dynamical equations, it is necessary to present the model used for the electrodynamic force acting upon the tether. The electrodynamic force which appears on a tether element, $F_{e d}^{d s}$, can be computed as:

$$
\overrightarrow{\boldsymbol{F}}_{e d}^{d s}=I(s, \overrightarrow{\boldsymbol{r}}, \overrightarrow{\boldsymbol{v}}, \overrightarrow{\boldsymbol{q}}, \dot{\overrightarrow{\boldsymbol{q}}}) \overrightarrow{\boldsymbol{u}}(\overrightarrow{\boldsymbol{q}}) \times \overrightarrow{\boldsymbol{B}}(s, \overrightarrow{\boldsymbol{r}})
$$

where $I(s, \overrightarrow{\boldsymbol{r}}, \overrightarrow{\boldsymbol{v}}, \overrightarrow{\boldsymbol{q}}, \dot{\overrightarrow{\boldsymbol{q}}})$ is the current along the tether, $\overrightarrow{\boldsymbol{B}}(s, \overrightarrow{\boldsymbol{r}})$ is the magnetic field at the tether element, $\overrightarrow{\boldsymbol{u}}(\overrightarrow{\boldsymbol{q}})$ represents the unit vector in the direction from the cathode to the anode, $s$ is the variable along the tether and $\overrightarrow{\boldsymbol{r}}, \overrightarrow{\boldsymbol{v}}, \overrightarrow{\boldsymbol{q}}, \dot{\overrightarrow{\boldsymbol{q}}}$ is the state of the system *. In order to simplify that expression, some hypothesis are made:

- Both the magnetic field and the velocity do not vary along the tether: $\overrightarrow{\boldsymbol{B}}(s, \overrightarrow{\boldsymbol{r}}) \approx \overrightarrow{\boldsymbol{B}}(\overrightarrow{\boldsymbol{r}})$, $\overrightarrow{\boldsymbol{v}}(s, \overrightarrow{\boldsymbol{r}}) \approx \overrightarrow{\boldsymbol{v}}(\overrightarrow{\boldsymbol{r}})$.

- The trajectory takes place in the equatorial plane and the tether motion is contained in this plane. Therefore, $\overrightarrow{\boldsymbol{u}}$ is a function only of an angle: $\phi$. Expressed in cylindrical coordinates: $\overrightarrow{\boldsymbol{u}}=\cos \phi \overrightarrow{\boldsymbol{u}}_{r}+\sin \phi \overrightarrow{\boldsymbol{u}}_{\theta}$. A scheme of the angles involved in the analysis can be seen in Figure 1.

\footnotetext{
${ }^{*} \vec{q}$ and $\dot{\vec{q}}$ stand for the variables used to represent the attitude of the tether.
} 
- The intensity along the tether is computed as the average in a given instant: $L_{t} I_{a v}(\overrightarrow{\boldsymbol{r}}, \overrightarrow{\boldsymbol{v}}, \phi)=$ $\int_{s_{0}}^{s_{f}} I(\xi, \overrightarrow{\boldsymbol{r}}, \overrightarrow{\boldsymbol{v}}, \phi) d \xi$.

- The magnetic field is a non titled dipole: $\overrightarrow{\boldsymbol{B}}(\overrightarrow{\boldsymbol{r}})=B(r) \overrightarrow{\boldsymbol{u}}_{z} *$

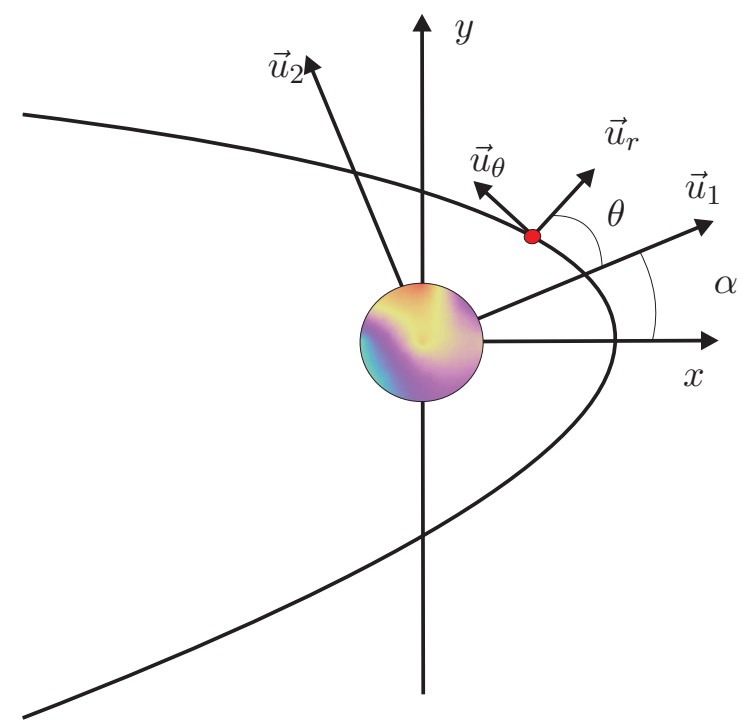

Figure 1. Angles and vectors considered in the jovian capture

Hence, the Lorentz force is written as:

$$
\overrightarrow{\boldsymbol{F}}_{e d}=L_{t} B(r) I_{a v}(\overrightarrow{\boldsymbol{r}}, \overrightarrow{\boldsymbol{v}}, \phi) \cdot\left(-\cos \phi \overrightarrow{\boldsymbol{u}}_{\theta}+\sin \phi \overrightarrow{\boldsymbol{u}}_{r}\right)
$$

The averaged intensity along the tether can be modeled as a function of the state of the system using the OML regime model as it is presented in Sanmartin et al. ${ }^{6}$. According to this model,

$$
I_{a v}(\overrightarrow{\boldsymbol{r}}, \overrightarrow{\boldsymbol{v}}, \phi)=\sigma_{c} E_{m}(\overrightarrow{\boldsymbol{r}}, \overrightarrow{\boldsymbol{v}}, \phi) d_{t} h_{t} i_{a v}\left(\ell_{t}(\overrightarrow{\boldsymbol{r}}, \overrightarrow{\boldsymbol{v}}, \phi), \Omega_{t}\right)
$$

where $\sigma_{c}$ is the conductivity of the tether material, $d_{t}$ and $h_{t}$ are the width and the thickness of the tape, respectively and $i_{a v}$ is a function of $\ell_{t}$ which is a non dimensional value proportional to the tether length and $\Omega_{t}$ which is a non dimensional value proportional to the electric load of the tether. The latter parameter is free and can be used as a control variable. In turn, $E_{m}$ is the motional electrical field projected on the tether direction, being its value:

$$
E_{m}(\overrightarrow{\boldsymbol{r}}, \overrightarrow{\boldsymbol{v}}, \phi)=\overrightarrow{\boldsymbol{u}} \cdot\left[\left(\overrightarrow{\boldsymbol{v}}-\overrightarrow{\boldsymbol{v}}_{p l}\right) \times \overrightarrow{\boldsymbol{B}}\right]=\overrightarrow{\boldsymbol{u}} \cdot\left[\overrightarrow{\boldsymbol{v}}^{r e l} \times \overrightarrow{\boldsymbol{B}}\right]=B(r)\left(v_{\theta}^{r e l} \cos \phi-v_{r}^{r e l} \sin \phi\right)
$$

Imposing the dipole moment description of the magnetic field:

$$
B(r)=-\mu_{m}\left(\frac{R_{4}}{r}\right)^{3}
$$

${ }^{*}$ The value of $B(r)$ in Jupiter will be negative, due to the dipole orientation. Nevertheless, as it will be showed later, the electrodynamic force is proportional to $B(r)^{2}$ and its sign only changes the direction of the intensity along the tether. 
the final result for the electrodynamic force as a function of the position, velocity and the angle $\phi$ ( $m_{t}=d_{t} h_{t} L_{t} / \rho_{v}$, tether mass) is:

$$
\overrightarrow{\boldsymbol{F}}_{e d}(\overrightarrow{\boldsymbol{r}}, \overrightarrow{\boldsymbol{v}}, \phi)=\frac{\sigma_{c} m_{t}}{\rho_{v}} \mu_{m}^{2} R_{4}^{6} \cdot \frac{i_{a v}}{r^{6}} \cdot\left(v_{\theta}^{r e l} \cos \phi-v_{r}^{r e l} \sin \phi\right) \cdot\left(-\cos \phi \overrightarrow{\boldsymbol{u}}_{\theta}+\sin \phi \overrightarrow{\boldsymbol{u}}_{r}\right)
$$

Nevertheless, we are interested in expressing the dynamics in terms of the osculating orbital elements. Moreover, the description must be valid for all the conics since the initial trajectory is a hyperbola and the final target trajectory is an ellipse. The radial and longitudinal velocity as well as the radius of all conics can be described as a function of the osculating orbit elements as follows:

$$
\overrightarrow{\boldsymbol{v}}=\frac{e \mu}{h} \sin \theta \overrightarrow{\boldsymbol{u}}_{r}+\frac{\mu}{h}(1+e \cos \theta) \overrightarrow{\boldsymbol{u}}_{\theta} \quad r=\frac{h^{2}}{\mu(1+e \cos \theta)}
$$

where $e$ is the eccentricity, $\theta$ the true anomaly and $h$ the specific angular momentum. For convenience, the specific angular momentum will be considered instead of the semi-major axis hereafter. Furthermore, if we take into account that the velocity of the plasma is $\overrightarrow{\boldsymbol{v}}_{p l}=r \Omega \overrightarrow{\boldsymbol{u}}_{\theta}{ }^{*}$, where $\Omega$ is the angular velocity of the sideral rotation of Jupiter. The final expression for the electrodynamic force acting on the tether is:

$$
\overrightarrow{\boldsymbol{F}}_{e d}=\Lambda i_{a v} \frac{\mathcal{P}_{1}(1+e \cos \theta)^{6}}{h^{13}}\left(-\cos \phi \overrightarrow{\boldsymbol{u}}_{\theta}+\sin \phi \overrightarrow{\boldsymbol{u}}_{r}\right)
$$

where

$$
\Lambda=\frac{m_{t} \mu_{4}^{7} \sigma_{c}}{\rho_{v}} \mu_{m}^{2} R_{4}^{6} \quad \text { and } \quad \mathcal{P}_{1}(\theta, h, \phi, e)=\cos \phi+e \cos (\theta+\phi)-\frac{\Omega h^{3} \cos \phi}{\mu_{\chi_{4}}^{2}(1+e \cos \theta)}
$$

\section{Dynamics}

The tether is considered to be rigid, therefore we should analyze the motion of a rigid body respect to a inertial frame $J x_{1} y_{1} z_{1}$. Let $\overrightarrow{\boldsymbol{r}}=\overrightarrow{\boldsymbol{r}}(t)$ be the position vector of the center of mass of the tether in the inertial reference frame $J x_{1} y_{1} z_{1}$. Hence, the equations of motion can be expressed as:

$$
M_{S / C} \ddot{\overrightarrow{\boldsymbol{r}}}=-\frac{M_{S / C} \mu_{4}}{|\overrightarrow{\boldsymbol{r}}|^{3}} \overrightarrow{\boldsymbol{r}}+\overrightarrow{\boldsymbol{F}}_{p}
$$

where $\overrightarrow{\boldsymbol{F}}_{p}$ is the perturbation force, defined as the resultant of all the external forces acting in the system except for the main term of the gravitational potential. We will consider the electrodynamic force as the only perturbation force $\overrightarrow{\boldsymbol{F}}_{p}=\overrightarrow{\boldsymbol{F}}_{e d}$. Under the assumptions enumerated in the previous paragraph, the Lorentz force has the form gathered in (7). Substituting the preceding expression in the equation of motion and expressing it in cylindric coordinates, we obtain:

$$
\begin{aligned}
M_{S / C}\left(\ddot{r}-r \dot{\theta}^{2}\right) & =-\frac{M_{S / C} \mu_{4}}{r^{2}}+\Lambda i_{a v} \frac{\mathcal{P}_{1}(1+e \cos \theta)^{6}}{h^{13}} \sin \phi \\
M_{S / C}(r \ddot{\theta}+2 \dot{r} \dot{\theta}) & =-\Lambda i_{a v} \frac{\mathcal{P}_{1}(1+e \cos \theta)^{6}}{h^{13}} \cos \phi
\end{aligned}
$$

${ }^{*}$ For prograde trajectories. 
This is a system of fourth order. The numerical integration of this system would provide us with the evolution of $r(t)$ and $\theta(t)$, given the functions $\Lambda$ and $\phi(t)$ and certain initial conditions. It can be noted that the attitude of the tether and the motion of the center of mass are not independent.

The goal of this section is to derive a complete set of equations of motion in terms of the osculating values of $h, e, \theta$ and $\alpha, \alpha$ being the longitude of the periapsis. In order to do that, we start by rewriting the acceleration of the center of mass of the tether:

$$
\frac{d \overrightarrow{\boldsymbol{v}}}{d t}=\left(-\frac{\mu_{4}}{r^{2}}+\frac{\Lambda i_{a v}}{M_{S C}} \frac{\mathcal{P}_{1}(1+e \cos \theta)^{6}}{h^{13}} \sin \phi\right) \overrightarrow{\boldsymbol{u}}_{r}-\frac{\Lambda i_{a v}}{M_{S C}} \frac{\mathcal{P}_{1}(1+e \cos \theta)^{6}}{h^{13}} \cos \phi \overrightarrow{\boldsymbol{u}}_{\theta}
$$

The time variation of the specific angular momentum can be obtained from its definition $\overrightarrow{\boldsymbol{h}}=\overrightarrow{\boldsymbol{r}} \times \overrightarrow{\boldsymbol{v}}$.

$$
\frac{d \overrightarrow{\boldsymbol{h}}}{d t}=\overrightarrow{\boldsymbol{r}} \times \frac{d \overrightarrow{\boldsymbol{v}}}{d t}=-\frac{r \Lambda i_{a v}}{M_{S C}} \frac{\mathcal{P}_{1}(1+e \cos \theta)^{6}}{h^{13}} \cos \phi \overrightarrow{\boldsymbol{u}}_{z}
$$

Substituting $r=h^{2} /(\mu(1+e \cos \theta))$, the final expression for the modulus of the derivative of the specific angular momentum is:

$$
\frac{d h}{d t}=-\frac{\Lambda i_{a v}}{\mu_{\psi_{4}} M_{S C}} \frac{\mathcal{P}_{1}(1+e \cos \theta)^{5}}{h^{11}} \cos \phi
$$

On the other hand, considering the definition of the eccentricity $\overrightarrow{\boldsymbol{e}}=-\overrightarrow{\boldsymbol{r}} / r-(\overrightarrow{\boldsymbol{h}} \times \overrightarrow{\boldsymbol{v}}) / \mu$, and taking its derivative with respect to time, we obtain:

$$
\frac{d \overrightarrow{\boldsymbol{e}}}{d t}=-\frac{\Lambda i_{a v}}{\mu_{\psi_{4}} M_{S C}} \frac{\mathcal{P}_{1}(1+e \cos \theta)^{6}}{h^{12}} \cos \phi\left[2 \overrightarrow{\boldsymbol{u}}_{r}+\left(\tan \phi-\frac{e \sin \theta}{1+e \cos \theta}\right) \overrightarrow{\boldsymbol{u}}_{\theta}\right]
$$

In the perifocal reference frame, the expression of the eccentricity is $\vec{e}=e \overrightarrow{\boldsymbol{u}}_{1}$, being:

$$
\overrightarrow{\boldsymbol{u}}_{r}=\cos \theta \overrightarrow{\boldsymbol{u}}_{1}+\sin \theta \overrightarrow{\boldsymbol{u}}_{2} \quad \overrightarrow{\boldsymbol{u}}_{\theta}=-\sin \theta \overrightarrow{\boldsymbol{u}}_{1}+\cos \theta \overrightarrow{\boldsymbol{u}}_{2}
$$

Differentiating the previous expression leads to:

$$
\frac{d \overrightarrow{\boldsymbol{e}}}{d t}=\frac{d e}{d t} \overrightarrow{\boldsymbol{u}}_{1}+e \frac{d \overrightarrow{\boldsymbol{u}}_{1}}{d t}
$$

Identifying equations (14) and (16), the result turns out to be:

$$
\begin{aligned}
\frac{d e}{d t} & =-\frac{\Lambda i_{a v}}{\mu_{4} M_{S C}} \frac{(1+e \cos \theta)^{6}}{h^{12}} \mathcal{P}_{1} \mathcal{P}_{2} \cos \phi \\
\frac{d \alpha}{d t} & =-\frac{\Lambda i_{a v}}{\mu_{4} M_{S C}} \frac{(1+e \cos \theta)^{6}}{h^{12}} \mathcal{P}_{1} \mathcal{P}_{3} \cos \phi \sin \theta
\end{aligned}
$$

where the functions $\mathcal{P}_{2}$ and $\mathcal{P}_{3}$ have been defined as:

$\mathcal{P}_{2}(\theta, \phi, e)=\left[2 \cos \theta+\frac{e \sin ^{2} \theta}{1+e \cos \theta}-\tan \phi \sin \theta\right] \quad \mathcal{P}_{3}(\theta, \phi, e)=\left[\frac{2}{e}+\frac{\tan \phi}{e \tan \theta}-\frac{\cos \theta}{1+e \cos \theta}\right]$ 
At last, it is necessary to establish the relation between true anomaly and time. Differentiating the expression of the velocity in cylindrical coordinates, we reach the formula for the variation of the true anomaly with time:

$$
\frac{d \theta}{d t}=\frac{\mu_{4}^{2}(1+e \cos \theta)^{2}}{h^{3}}-\frac{\Lambda i_{a v}(1+e \cos \theta)^{6}}{\mu_{4} M_{S / C} h^{12}} \mathcal{P}_{1} \mathcal{P}_{4}
$$

where the function $\mathcal{P}_{4}$ is defined as follows:

$$
\mathcal{P}_{4}(\theta, \phi, e)=\left[\sin \phi+\cos \phi \tan \theta\left(\frac{2+e \cos \theta}{1+e \cos \theta}-(e+\cos \theta)\left(\frac{2}{e}+\frac{\tan \phi}{e \tan \theta}-\cos \theta\right)\right)\right]
$$

Gathering the results in a system of equations, we have a new system of fourth order:

$$
\begin{aligned}
\frac{d h}{d t} & =-\frac{\Lambda i_{a v}}{\mu_{4} M_{S C}} \frac{\mathcal{P}_{1}(1+e \cos \theta)^{5}}{h^{11}} \cos \phi \\
\frac{d e}{d t} & =-\frac{\Lambda i_{a v}}{\mu_{4} M_{S C}} \frac{(1+e \cos \theta)^{6}}{h^{12}} \mathcal{P}_{1} \mathcal{P}_{2} \cos \phi \\
\frac{d \alpha}{d t} & =-\frac{\Lambda i_{a v}}{\mu_{4} M_{S C}} \frac{(1+e \cos \theta)^{6}}{h^{12}} \mathcal{P}_{1} \mathcal{P}_{3} \cos \phi \sin \theta \\
\frac{d \theta}{d t} & =\frac{\mu_{\psi_{4}}^{2}(1+e \cos \theta)^{2}}{h^{3}}-\frac{\Lambda i_{a v}(1+e \cos \theta)^{6}}{\mu_{4} M_{S / C} h^{12}} \mathcal{P}_{1} \mathcal{P}_{4}
\end{aligned}
$$

Non Dimensional Formulation The utilization of non dimensional equations instead of the dimensional ones is desirable since it allows to define the main parameters involved in the capture process. We will use $h_{c}=h_{0}$, the initial value of the specific angular momentum, as its characteristic value. Regarding the characteristic time, there are two possible choices, in one hand, the angular velocity of the sideral rotation of Jupiter, $\Omega$, and on the other hand, the time related to the orbital motion, $\tau_{c}=h_{0}^{3} / \mu_{2}^{2}$. We will use $\tau_{c}$ as the characteristic time calling $\gamma$ the relation between both times: $\gamma=\Omega \tau_{c}$. Using that variables, the non dimensional equations of motion can be expressed as follows:

$$
\begin{aligned}
\frac{d \hat{h}}{d \tau} & =-\hat{\Lambda} i_{a v} \frac{(1+e \cos \theta)^{5}}{\hat{h}^{11}} \mathcal{P}_{1} \cos \phi \\
\frac{d e}{d \tau} & =-\hat{\Lambda} i_{a v} \frac{(1+e \cos \theta)^{6}}{\hat{h}^{12}} \mathcal{P}_{1} \mathcal{P}_{2} \cos \phi \\
\frac{d \alpha}{d \tau} & =-\hat{\Lambda} i_{a v} \frac{(1+e \cos \theta)^{6}}{\hat{h}^{12}} \mathcal{P}_{1} \mathcal{P}_{3} \cos \phi \sin \theta \\
\frac{d \theta}{d \tau} & =\frac{(1+e \cos \theta)^{2}}{\hat{h}^{3}}-\hat{\Lambda} i_{a v} \frac{(1+e \cos \theta)^{6}}{\hat{h}^{12}} \mathcal{P}_{1} \mathcal{P}_{4}
\end{aligned}
$$

where

$$
\hat{\Lambda}=\frac{m_{t}}{M_{S C}} \frac{\sigma_{c}}{\rho_{v}} \frac{\mu_{m}^{2} R_{4}^{6}}{\tau_{c}^{2} h_{0}^{3}}
$$

is a non dimensional value. The initial conditions needed to integrate the system (24-27) are:

$$
\hat{h}(0)=1 ; \quad e(0)=e_{0} ; \quad \alpha(0)=0
$$


And the initial true anomaly fulfilling the constraint (from $E_{m}=0$ ):

$$
\mathcal{P}_{1}=0 \Rightarrow\left(\cos \phi+e_{0} \cos \left(\theta_{0}+\phi\right)\right)\left(1+e_{0} \cos \theta_{0}\right)=\gamma \cos \phi
$$

In some situations, it can be useful to express the system as a function of the true anomaly instead of the non dimensional time, since that allows to restrict the integration to the drag arc. As $\tau$ does not appear explicitly in the dynamic equations, the true anomaly will be used as the independent variable using the relation:

$$
d \tau=\frac{\hat{h}^{12} d \theta}{(1+e \cos \theta)^{2}\left[\hat{h}^{9}+\hat{\Lambda} i_{a v}(1+e \cos \theta)^{4} \mathcal{P}_{1} \mathcal{P}_{4}\right]}
$$

This formula can be used provided $d \theta / d \tau$ is not canceled. This situation is extreme, undesirable and unlikely to happen. Eventually, the final expression for the dynamic equations is found to be:

$$
\begin{aligned}
\frac{d \hat{h}}{d \theta} & =-\hat{\Lambda} i_{a v} \frac{\hat{h}(1+e \cos \theta)^{3} \mathcal{P}_{1} \cos \phi}{\hat{h}^{9}-\hat{\Lambda} i_{a v}(1+e \cos \theta)^{4} \mathcal{P}_{1} \mathcal{P}_{4}} \\
\frac{d e}{d \theta} & =-\hat{\Lambda} i_{a v} \frac{(1+e \cos \theta)^{4} \mathcal{P}_{1} \mathcal{P}_{2} \cos \phi}{\hat{h}^{9}-\hat{\Lambda} i_{a v}(1+e \cos \theta)^{4} \mathcal{P}_{1} \mathcal{P}_{4}} \\
\frac{d \alpha}{d \theta} & =-\hat{\Lambda} i_{a v} \frac{(1+e \cos \theta)^{4} \mathcal{P}_{1} \mathcal{P}_{3} \cos \phi \sin \theta}{\hat{h}^{9}-\hat{\Lambda} i_{a v}(1+e \cos \theta)^{4} \mathcal{P}_{1} \mathcal{P}_{4}}
\end{aligned}
$$

With the initial conditions:

$$
\hat{h}\left(\theta_{0}\right)=1 ; \quad e\left(\theta_{0}\right)=e_{0} ; \quad \alpha\left(\theta_{0}\right)=0
$$

\section{ATTITUDE DYNAMICS}

From the expressions obtained in the previous section, it can be noted that the attitude plays an important role in the motion of the center of mass. It is, therefore, compulsory to study the attitude behavior of the tethered systems. This paragraph is intended to present the attitude dynamics in a comprehensive way considering two possible situations: non rotating and rotating tethers.

\section{Non Rotating Tethers}

The equation which governs the attitude dynamics is the equation of the angular momentum

$$
\frac{d \overrightarrow{\boldsymbol{L}}_{G}}{d t}=\overrightarrow{\boldsymbol{M}}
$$

where the angular momentum can be expressed as a function of the angular velocity and the inertia

tensor: $\overrightarrow{\boldsymbol{L}}_{G}=\overline{\bar{I}}_{G} \circ \overrightarrow{\boldsymbol{\omega}}$. In turn, the angular velocity is related to the unitary vector $\overrightarrow{\boldsymbol{u}}$ in the tether direction, assuming the tether is a rigid body and remains straight: $\overrightarrow{\boldsymbol{\omega}}=\overrightarrow{\boldsymbol{u}} \times \dot{\vec{u}}+(\overrightarrow{\boldsymbol{\omega}} \cdot \overrightarrow{\boldsymbol{u}}) \overrightarrow{\boldsymbol{u}}$. Therefore, the equation (33) can then be rewritten as:

$$
\overrightarrow{\boldsymbol{u}} \times \ddot{\vec{u}}=\frac{1}{I_{s}} \overrightarrow{\boldsymbol{M}}
$$


The torques considered in this work will be the gravity gradient and the electrodynamic torque. The momentum due to the gravity forces is analyzed in first place. It can be established as:

$$
\overrightarrow{\boldsymbol{M}}_{g g}=-\int_{\Omega_{0}}\left(\overrightarrow{\boldsymbol{r}}-\overrightarrow{\boldsymbol{r}}_{G}\right) \times \frac{\mu_{4}}{r^{3}} \overrightarrow{\boldsymbol{r}} d m=\overrightarrow{\boldsymbol{r}}_{G} \times \int_{\Omega_{0}} \frac{\mu_{4}}{r^{3}} \overrightarrow{\boldsymbol{r}} d m
$$

where $\overrightarrow{\boldsymbol{r}}$ and $\overrightarrow{\boldsymbol{r}}_{G}$ are the position vector of each mass element and the center of mass of the tether, respectively, and $\Omega_{0}$ is the volume of the whole system. Considering $\overrightarrow{\boldsymbol{r}}=\overrightarrow{\boldsymbol{r}}_{G}+\overrightarrow{\boldsymbol{s}}$ with $|\overrightarrow{\boldsymbol{s}}| \ll\left|\overrightarrow{\boldsymbol{r}}_{G}\right|$, the first approximation of the gravity gradient is as follows:

$$
\overrightarrow{\boldsymbol{M}}_{g g}=\overrightarrow{\boldsymbol{r}}_{G} \times \int_{\Omega_{0}} \frac{\mu_{4}}{\left|\overrightarrow{\boldsymbol{r}}_{G}+\overrightarrow{\boldsymbol{s}}\right|^{3}} \overrightarrow{\boldsymbol{s}} d m \approx \frac{3 \mu_{4}}{r_{G}^{5}} \overrightarrow{\boldsymbol{r}}_{G} \times\left(\overline{\overline{\mathbf{I}}}_{G} \circ \overrightarrow{\boldsymbol{r}}_{G}\right)
$$

Expressing the vector $\overrightarrow{\boldsymbol{u}}$ in the inertial reference frame: $\overrightarrow{\boldsymbol{u}}=\cos \psi \overrightarrow{\boldsymbol{i}}+\sin \psi \overrightarrow{\boldsymbol{j}}$, the vector product $\overrightarrow{\boldsymbol{u}} \times \ddot{\vec{u}}$ is a function of the angle $\psi$ :

$$
\overrightarrow{\boldsymbol{u}} \times \ddot{\overrightarrow{\boldsymbol{u}}}=\ddot{\psi} \overrightarrow{\boldsymbol{k}}=\frac{1}{I_{s}} \overrightarrow{\boldsymbol{M}}
$$

On the other hand, naming $\overrightarrow{\boldsymbol{r}}_{G}=r_{G} \overrightarrow{\boldsymbol{u}}_{r}$, the identity $\overrightarrow{\boldsymbol{u}}_{r}=\left(\overrightarrow{\boldsymbol{u}}_{r} \cdot \overrightarrow{\boldsymbol{u}}\right) \overrightarrow{\boldsymbol{u}}+\overrightarrow{\boldsymbol{u}} \times\left(\overrightarrow{\boldsymbol{u}}_{r} \times \overrightarrow{\boldsymbol{u}}\right)$ allows to write:

$$
\overline{\overline{\mathbf{I}}}_{G} \circ \overrightarrow{\boldsymbol{u}}_{r}=I_{s} \overrightarrow{\boldsymbol{u}} \times\left(\overrightarrow{\boldsymbol{u}}_{r} \times \overrightarrow{\boldsymbol{u}}\right)=I_{s}\left\{\overrightarrow{\boldsymbol{u}}_{r}-\overrightarrow{\boldsymbol{u}}\left(\overrightarrow{\boldsymbol{u}}_{r} \cdot \overrightarrow{\boldsymbol{u}}\right)\right\}
$$

The gravity torque can be, therefore, expressed as:

$$
\overrightarrow{\boldsymbol{M}}_{g g}=\frac{3 \mu_{4}}{r_{G}^{3}} I_{s}\left(\overrightarrow{\boldsymbol{u}} \times \overrightarrow{\boldsymbol{u}}_{r}\right)\left(\overrightarrow{\boldsymbol{u}}_{r} \cdot \overrightarrow{\boldsymbol{u}}\right)
$$

Taking into account that $\overrightarrow{\boldsymbol{u}}_{r}=\cos (\alpha+\theta) \overrightarrow{\boldsymbol{i}}+\sin (\alpha+\theta) \overrightarrow{\boldsymbol{j}}$, the final expression for the equation of the angular momentum is stated as:

$$
\ddot{\psi}=\frac{3 \mu_{4}}{r_{G}^{3}} \sin (\theta+\alpha-\psi) \cos (\theta+\alpha-\psi)
$$

In terms of the angle $\phi$ (related to the rotating radial frame), using the identity $\psi=\phi+\alpha+\theta$, the equations take the form:

$$
\ddot{\phi}=-(\ddot{\theta}+\ddot{\alpha})-\frac{3 \mu_{4}}{r_{G}^{3}} \sin \phi \cos \phi
$$

On the other hand, the electrodynamic torque due to the Lorentz force acting over the tether can be computed as:

$$
\overrightarrow{\boldsymbol{M}}_{e d}=\int_{\Omega}\left(s \overrightarrow{\boldsymbol{u}} \times \overrightarrow{\boldsymbol{F}}_{e d}^{d s}\right) d s
$$

Considering the previous definition of the electrodynamic force (7) and assuming the same hypothesis used to simplified its expression in the previous analysis, we obtain:

$$
\overrightarrow{\boldsymbol{M}}_{e d}=\overrightarrow{\boldsymbol{u}} \times(\overrightarrow{\boldsymbol{u}} \times \overrightarrow{\boldsymbol{B}}) \int_{\Omega} s I d s
$$

With respect to the derivation of the force, there is a slight difference regarding the intensity, since the value of the integral is no longer the average value but the first momentum of the current profile.

$$
L_{t}^{2} I_{m 1}=\int_{\Omega} s I d s \quad \text { dimensional } \quad I_{m 1}=\sigma_{c} E_{m} d_{t} h_{t} i_{m 1}\left(\ell_{t}\right) \quad \text { non dimensional }
$$


That yields the following relation:

$$
\overrightarrow{\boldsymbol{M}}_{e d}=-B(r)^{2} \frac{\sigma_{c} m_{t} L_{t}}{\rho_{v}} i_{m 1}\left(v_{\theta}^{r e l} \cos \phi-v_{r}^{r e l} \sin \phi\right) \overrightarrow{\boldsymbol{u}}_{z}
$$

The value of the moment of inertia as a function of the parameters of the geometry of mass is gathered in (2). Introducing this expression and the term of the electrodynamic torque in the attitude equation, we finally obtain:

$$
\begin{array}{r}
\ddot{\psi}=-\frac{3 \mu_{\psi}}{r_{G}^{3}} \sin (\psi-\theta-\alpha) \cos (\psi-\theta-\alpha)-\frac{12 B(r)^{2}}{L_{t}\left(3 \sin ^{2}(2 \chi)-2 \Gamma\right)} \frac{\sigma_{c}}{\rho_{v}} \\
\cdot i_{m 1}\left(v_{\theta}^{r e l} \cos (\psi-\theta-\alpha)-v_{r}^{r e l} \sin (\psi-\theta-\alpha)\right)
\end{array}
$$

As it was made for the motion of the center of mass, the equation of the attitude dynamics can be expressed in non dimensional form. Using the same characteristic magnitudes, the results are gathered in the following lines. Describing the attitude by means of the inertial angle $\psi$ :

$$
\frac{d^{2} \psi}{d \tau^{2}}=-\frac{3}{2} \frac{(1+e \cos \theta)^{3}}{\hat{h}^{6}} \sin (2(\psi-\theta-\alpha))-\frac{\kappa i_{m 1}}{\left(3 \sin ^{2}(2 \chi)-2 \Gamma\right)} \frac{(1+e \cos \theta)^{6}}{\hat{h}^{13}} \mathcal{P}_{1}
$$

or in terms of the angle $\phi$ :

$$
\frac{d^{2} \phi}{d \tau^{2}}=-\frac{d^{2} \theta}{d \tau^{2}}-\frac{d^{2} \alpha}{d \tau^{2}}-\frac{3}{2} \frac{(1+e \cos \theta)^{3}}{\hat{h}^{6}} \sin (2 \phi)-\frac{\kappa i_{m 1}}{\left(3 \sin ^{2}(2 \chi)-2 \Gamma\right)} \frac{(1+e \cos \theta)^{6}}{\hat{h}^{13}} \mathcal{P}_{1}
$$

where

$$
\kappa=\frac{12 \mu_{m}^{2} R_{4}^{6} \sigma_{c} \mu_{4}^{3}}{L_{t} h_{0}^{7} \rho_{v}}
$$

\section{Rotating Tethers}

The attitude equations which govern the motion of the rotating tethers are the same as we have already seen in the previous paragraph for non rotating tethers. Nevertheless, it is possible to perform a qualitative analysis of their behavior as a function of its period of rotation $T_{\text {rot }}$, which constitutes another dynamical characteristic time. Depending on the ratio $\tau_{c} / T_{r o t}$, the possible cases are between two extremes: slowly rotating tethers $\tau_{c} \ll T_{\text {rot }}$ and rapidly rotating tethers $\tau_{c} \gg T_{\text {rot }}$. The former can be handled assuming the tether is non rotating and using the relations gathered in the previous paragraph. In the latter, some considerations can be made about the nature of the attitude motion.

The constraint that must be fulfilled to apply the fast rotating tether approximation, $\tau_{c} \ll T_{\text {rot }}$, is equivalent to assume that the angular velocity in non dimensional variables is $d \psi / d \tau \gg 2 \pi$, since

$$
T_{\text {rot }} \frac{d \psi}{d t}=2 \pi \Rightarrow \frac{\tau_{c}}{T_{\text {rot }}}=\frac{1}{2 \pi} \frac{d \phi}{d \tau}
$$

On the other hand, due to the orders of magnitude of the derivatives of the state variables are:

$$
\frac{d \hat{h}}{d \tau}, \frac{d e}{d \tau}, \frac{d \alpha}{d \tau} \sim \hat{\Lambda} \quad \frac{d \theta}{d \tau} \sim \mathcal{O}(1)
$$


the relation among the derivatives respect to time of the variables responds to

$$
\frac{d \psi}{d \tau} \gg \frac{d \hat{h}}{d \tau}, \frac{d e}{d \tau}, \frac{d \alpha}{d \tau}, \frac{d \theta}{d \tau}
$$

In this situation we can assume that $\frac{d \psi}{d \tau}=\frac{d(\theta+\alpha+\phi)}{d \tau} \approx \frac{d \phi}{d \tau}$ and $d \phi / d \tau$ is nearly constant in the interval of time $\Delta \tau=\pi /(d \phi / d \tau)$. Hence, an average in the tether orientation angle will equal the time average ${ }^{6}$ in the interval $\Delta \tau$ and the state variables are approximately constant during that interval. All these considerations yield to the formulation of the averaged attitude equations for fast rotating tethers.

$$
<\frac{d^{2} \psi}{d \tau^{2}}>=-\frac{\kappa}{3 \sin ^{2}(2 \chi)-2 \Gamma} \frac{(1+e \cos \theta)^{6}}{\hat{h}^{13}} \frac{1}{\Delta \psi} \int_{\psi_{0}}^{\psi_{f}} \mathcal{P}_{1}(\varphi) i_{m 1}(\varphi) d \varphi
$$

where $<>$ stands for the average value in a revolution, while the initial, $\psi_{0}$, and final, $\psi_{f}$, values of the integrals correspond to the angles where the value of $E_{m}$ vanishes, which is equivalent to the solutions of the non linear equation $\mathcal{P}_{1} \equiv 0$. Besides, the value of $\Delta \psi$ is equal to $\psi_{f}-\psi_{0}$, and providing $\mathcal{P}_{1}(\psi, \hat{h}, e, \alpha, \theta ; \gamma)=0 \Rightarrow \mathcal{P}_{1}(\psi+\pi, \hat{h}, e, \alpha, \theta ; \gamma)=0$ we will have $\Delta \psi=\pi$.

Therefore, the variation in the angular velocity of fast rotating tethers depends only on the electrodynamic torque, i.e., the geometry of mass of the system as well as the tether current profile. In Sanmartin et al. ${ }^{6}$, a similar result is achieved, drawing an interesting conclusion for a tether with equal end masses and no electric load: the Lorentz torque is continually antiparallel to the spin of Jupiter, and, consequently, the tether should enter the drag arc with a negative spin respect to the Jupiter angular velocity since, otherwise, the Lorentz torque could brake the tether spin preventing the tether to have the suitable attitude to perform the capture.

Averaged Equations For fast rotating tethers, the integration of the system of equations (24-27) along with (43) leads to a stiff problem with two extremely different characteristic times. In order to deal with this situation, the averaged equations of the motion of the center of mass can be derived in the same way as the attitude equations for this kind of systems, providing the conditions (45) are fulfilled.

$$
\begin{aligned}
<\frac{d \hat{h}}{d \tau}>=-\frac{\hat{\Lambda}(1+e \cos \theta)^{5}}{\pi \hat{h}^{11}} \mathcal{Q}_{1} \\
<\frac{d e}{d \tau}>=-\frac{\hat{\Lambda}(1+e \cos \theta)^{6}}{\pi \hat{h}^{12}}\left[\left(2 \cos \theta+\frac{e \sin ^{2} \theta}{1+e \cos \theta}\right) \mathcal{Q}_{1}-\sin \theta \mathcal{Q}_{2}\right] \\
<\frac{d \alpha}{d \tau}>=-\frac{\hat{\Lambda}(1+e \cos \theta)^{6}}{\pi \hat{h}^{12}}\left[\sin \theta\left(\frac{2}{e}-\frac{\cos \theta}{1+e \cos \theta}\right) \mathcal{Q}_{1}+\frac{\cos \theta}{e} \mathcal{Q}_{2}\right] \\
<\frac{d \theta}{d \tau}>=\frac{(1+e \cos \theta)^{2}}{\hat{h}^{3}}-\frac{\hat{\Lambda}(1+e \cos \theta)^{6}}{\pi \hat{h}^{12}} \\
\quad\left[\cos \theta\left(\frac{2+e \cos \theta}{1+e \cos \theta}-(e+\cos \theta)\left(\frac{2}{e}-\cos \theta\right)\right) \mathcal{Q}_{1}-\frac{\cos \theta}{e} \mathcal{Q}_{2}\right]
\end{aligned}
$$


Where the following notation have been used:

$$
\begin{array}{ll}
\mathcal{Q}_{1}=\mathcal{I}_{1}\left(1-\frac{\gamma \hat{h}^{3}}{1+e \cos \theta}\right)+e \mathcal{I}_{2} & \mathcal{Q}_{2}=\mathcal{I}_{3}\left(1-\frac{\gamma \hat{h}^{3}}{1+e \cos \theta}\right)+e \mathcal{I}_{4} \\
\mathcal{I}_{1}=\int_{\psi_{0}}^{\psi_{f}} i_{a v} \cos ^{2}(\varphi-\theta-\alpha) d \varphi & \mathcal{I}_{2}=\int_{\psi_{0}}^{\psi_{f}} i_{a v} \cos (\varphi-\alpha) \cos (\varphi-\theta-\alpha) d \varphi \\
\mathcal{I}_{3}=\int_{\psi_{0}}^{\psi_{f}} i_{a v} \cos (\varphi-\theta-\alpha) \sin (\varphi-\theta-\alpha) d \varphi & \mathcal{I}_{4}=\int_{\psi_{0}}^{\psi_{f}} i_{a v} \cos (\varphi-\alpha) \sin (\varphi-\theta-\alpha) d \varphi
\end{array}
$$

To compute their value it is necessary to solve the non linear equation $\mathcal{P}_{1}(\hat{h}, e, \theta ; \phi) \equiv 0$ in each position determined by $(\hat{h}, e, \theta)$. The condition $\Delta \psi=\psi_{f}-\psi_{0}=\pi$ has been taken into account as well.

\section{CONTROL ON NON ROTATING TETHERS}

While for rotating tethers attitude is not an important issue, as it has been seen before, for non rotating tethers this constitutes a matter of the first relevance. That is due to the fact that having the right orientation is compulsory to complete the capture manoeuvre successfully. In this section, the feasibility of obtaining the desired attitude without using external means is explored.

\section{Assessment of Control Authority}

The first question addressed is whether or not the electrodynamic torque is able to keep the attitude of the tether controlled. Therefore, the required control torque and the operational limits of the electrodynamic torque will be analyzed to eventually compare the both of them.

Needed Torque The attitude dynamics are governed by equation (44) in terms of the angle $\phi$. To evaluate the torque needed to maintain a prefigured attitude profile, we will consider the function:

$\mathcal{G}(\tau)=-\left(\frac{d^{2} \phi}{d \tau^{2}}+\frac{d^{2} \theta}{d \tau^{2}}+\frac{d^{2} \alpha}{d \tau^{2}}+\frac{3}{2} \frac{(1+e \cos \theta)^{3}}{\hat{h}^{6}} \sin (2 \phi)\right) \frac{\kappa \hat{h}^{13}(\tau)}{(1+e(\tau) \cos \theta(\tau))^{6} \mathcal{P}_{1}(\hat{h}(\tau), e(\tau), \theta(\tau))}$

According to the equation (44) the needed torque can be written as:

$$
i_{m 1}^{\text {need }}=\left(3 \sin ^{2}(2 \chi)-2 \Gamma\right) \mathcal{G}(\tau)
$$

The function $\mathcal{G}(\tau)$ can be obtained as a result of the integration of the equations of motion for a given attitude profile $\phi(\tau)$. It should be noted that this expression is only valid for $\mathcal{P}_{1} \geq 0$, i.e., only along the arc where it is possible to generate drag. On the other hand, $\chi$ and $\Gamma$ are functions of the geometry of mass of the system. And consequently, the function $\mathcal{F}_{I_{s}}=\left(3 \sin ^{2}(2 \chi)-2 \Gamma\right)$ is easily computed given the values of the end masses $m_{1}$ and $m_{2}$ and the tether mass $m_{t}$. The values corresponding the self-balance configurations (with $\chi \in \sim\left[40^{\circ}, 50^{\circ}\right]$ ) vary between 1 and 3 . Accordingly, the influence of this factor is not expected to be determinant in the controllability.

On the other hand, the function $\mathcal{G}(\tau)$ must be computed as a result of the integration of the equations of motion. A reference case is chosen to perform the above-mentioned integration, characterized by the following parameters: 


\begin{tabular}{|l|c|}
\hline & \\
Periapsis radius (initial hyperbolic trajectory) & $1.42 R_{4}$ \\
& $5.64 \mathrm{~km} / \mathrm{s}$ \\
\hline Tether length & $100 \mathrm{~km}$ \\
Tether thickness & $0.05 \mathrm{~mm}$ \\
Tether mass & $405 \mathrm{~kg}$ \\
Spacecraft mass & $905 \mathrm{~kg}$ \\
Mass angle $\chi$ & $40^{\circ}$ \\
Tether material & Aluminium \\
\hline
\end{tabular}

Additionally, an attitude profile should be established. We will consider three cases regarding the suitability from the point of view of the electrodynamic forces which can be generated.

- $\phi=0$. The tether is pointing continuously toward Jupiter.

- $\psi=0$. The tether keeps a fix inertial orientation.

- $\overrightarrow{\boldsymbol{u}} \cdot \overrightarrow{\boldsymbol{v}}^{r e l}=0$. The tether is forced to be perpendicular to both the relative velocity to the plasma and the magnetic field.

The results obtained are shown in the last paragraph of this section.

Electrodynamic Torque In the equation (51), the left term depends on the intensity profile along the tether. In fact, $i_{m 1}$ is the non dimensional first order moment of the intensity respect to the center of mass, i.e.:

$$
i_{m 1}=\int_{0}^{\ell_{t}}\left(\xi_{G}-\xi\right) i(\xi) d \xi=\ell_{t} \cos ^{2}(\chi) U_{1}\left(\ell_{t}, \Omega_{t}\right)-U_{2}\left(\ell_{t}, \Omega_{t}\right)
$$

where: $U_{1}\left(\ell_{t}, \Omega_{t}\right)=\int_{0}^{\ell_{t}} i(\xi) d \xi$ and $U_{2}\left(\ell_{t}, \Omega_{t}\right)=\int_{0}^{\ell_{t}} \xi i(\xi) d \xi$. To compute the values of $U_{1}$ and $U_{2}$, it is necessary to solve the two boundary value problem for the intensity $i(\xi)$ as it is exposed in Peláez and Sanjurjo-Rivo ${ }^{13}$. With that formulation, $U_{1}$ and $U_{2}$ are:

$$
\begin{aligned}
U_{1}\left(\ell_{t}, \Omega_{t}\right) & =\ell_{t}+\varphi_{C}-\varphi_{A} \\
U_{2}\left(\ell_{t}, \Omega_{t}\right) & =\varphi_{C} \ell_{t}+\frac{\ell_{t}^{2}}{2}-\frac{4}{3}\left(1-i_{B}\right)^{5 / 3}\left[\int_{0}^{v_{0}} \sinh ^{5 / 3}(\zeta) d \zeta-\frac{1}{\mu^{4 / 3}} \int_{0}^{v_{T}} \sinh ^{5 / 3}(\zeta) d \zeta\right]
\end{aligned}
$$

We are interested on the operational limits of the control, being $\Omega_{t}=0$ and $\Omega_{t} \rightarrow \infty$. In the first case, $\Omega_{t}=0$, we have $\varphi_{C}=0, \ell_{t}=\xi_{B}$ and therefore:

$$
\begin{aligned}
& U_{1}\left(\ell_{t}, 0\right)=\ell_{t}-\varphi_{A} \\
& U_{2}\left(\ell_{t}, 0\right)=\frac{\ell_{t}^{2}}{2}-\frac{4}{3}\left(1-i_{B}\right)^{5 / 3} \int_{0}^{v_{0}} \sinh ^{5 / 3}(\zeta) d \zeta
\end{aligned}
$$

In the second case, we have $i_{C}=0$ and $v_{T}=v_{0}$, and then:

$$
\begin{aligned}
U_{1}\left(\ell_{t}, \rightarrow \infty\right) & =\ell_{t}+\varphi_{C}-\varphi_{A} \\
U_{2}\left(\ell_{t}, \rightarrow \infty\right) & =\frac{\ell_{t}^{2}}{2}+\varphi_{C} \ell_{t}-\frac{4}{3}\left(1-i_{B}\right)^{5 / 3} \int_{0}^{v_{0}} \sinh ^{5 / 3}(\zeta) d \zeta\left(1-\frac{1}{\mu^{4 / 3}}\right)
\end{aligned}
$$

In the next paragraph the limits $i_{m 1}\left(\Omega_{t}=0\right)$ and $i_{m 1}\left(\Omega_{t} \rightarrow \infty\right)$ are plotted in the same graphs as the needed values $i_{m 1}^{\text {need }}$ as functions of $\ell_{t}$ and $\tau$. 
Comparison In the follow graphics we compare the needed values of the electrodynamic torque and its limits of operation. The needed values are represented by blue crosses while the limits of operation appear as black lines in Figure 2, 3 and 4.
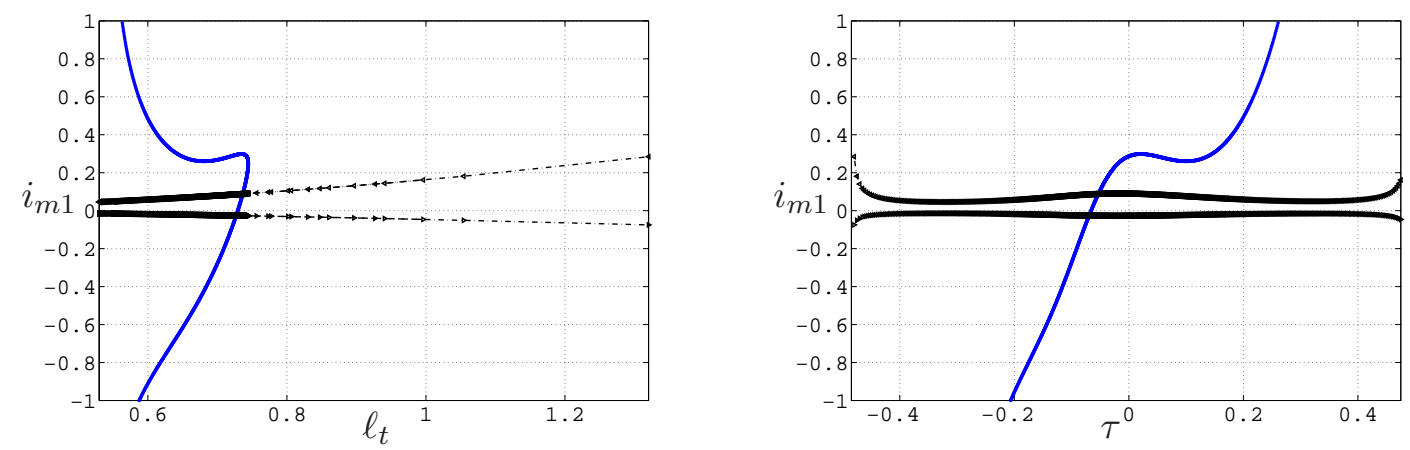

Figure 2 Needed values of $i_{m 1}$ as a function of $\ell_{t}$ during the capture (blue crosses) and the control limits of $i_{m 1}$ (black lines). Control law: $\phi \equiv 0$
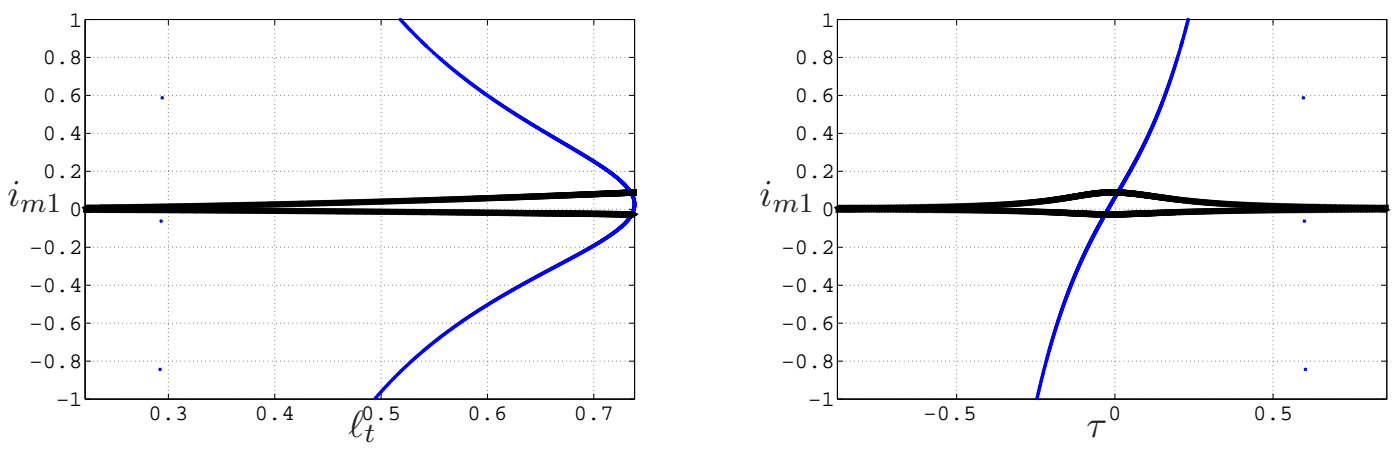

Figure 3 Needed values of $i_{m 1}$ as a function of $\ell_{t}$ during the capture (blue crosses) and the control limits of $i_{m 1}$ (black lines). Control law: $\phi \equiv 0$

We can conclude that none of the control laws can provide the needed torque to keep the prefigured attitude while the electrodynamic tether is operative. Furthermore, the electrodynamic torque is only available during the drag arc, and therefore there is no compensating torque outside the drag sphere.

\section{Control Solution}

From the results exposed in Scheeres et al. ${ }^{14}$ and Scheeres ${ }^{15}$, the change in angular momentum during an equatorial flyby will only have a component along the z-axis proportional to $\sin 2(\omega+\Omega)$. We can cancel this term by means of making the $x$-axis of the tether fixed reference frame at the periapsis point towards the center of mass of the planet, where the $\mathrm{x}$-axis in the body fixed reference frame is defined to lie along the axis of minimum momentum of inertia. That is, the tether should be aligned with the local vertical in the periapsis passage.

As a first step we will analyze the motion of the tethered system when the electrodynamic forces are not present. Our objective will be to obtain a solution which fulfill the condition of keeping 

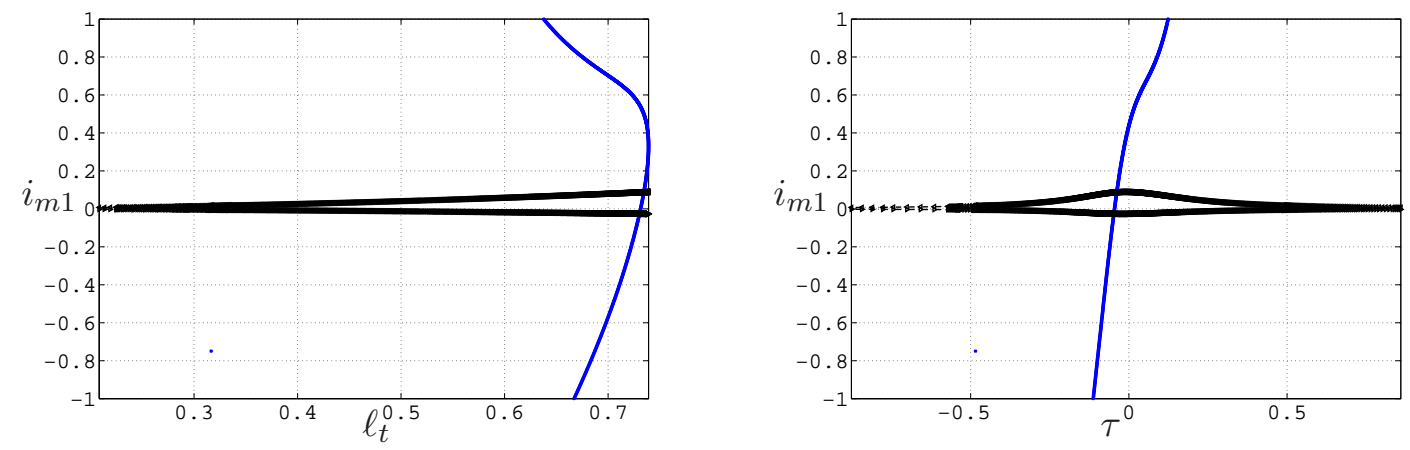

Figure 4 Needed values of $i_{m 1}$ as a function of $\ell_{t}$ during the capture (blue crosses) and the control limits of $i_{m 1}$ (black lines). Control law: $\overrightarrow{\boldsymbol{v}}^{\text {rel }} \cdot \overrightarrow{\boldsymbol{u}}$

the tether without spin at the end of the flyby. The non dimensional form of the equation which governs the attitude in terms of a inertial angle, $\psi$ is (43). To get the necessary initial condition in the angle $\psi$ to keep the tether non rotating, the equation (43) should be solved with these boundary conditions:

$$
\begin{aligned}
\dot{\psi}\left(\theta_{0}\right) & =0 \\
\psi(\theta=0) & =0
\end{aligned}
$$

One relevant issue in this approach is where the initial condition is considered to be. Since the angle which defines the asymptote of the hyperbola is $\theta_{a}=\arccos (-1 / e)$, where $e$ is its eccentricity, the initial angle $\theta_{0}$ must be a fraction of $\theta_{a}$. The solution can vary slightly when different values of $\theta_{0}$ are considered. When we take $\theta_{0}=0.99 \theta_{a}$, the initial angle $\psi_{0}$ which provides no change of the angular momentum is: $\psi_{0} \approx-57.2^{\circ}$. In Figure 5 the solution along the whole trajectory is represented in terms of the inertial angle and the non dimensional angular velocity.
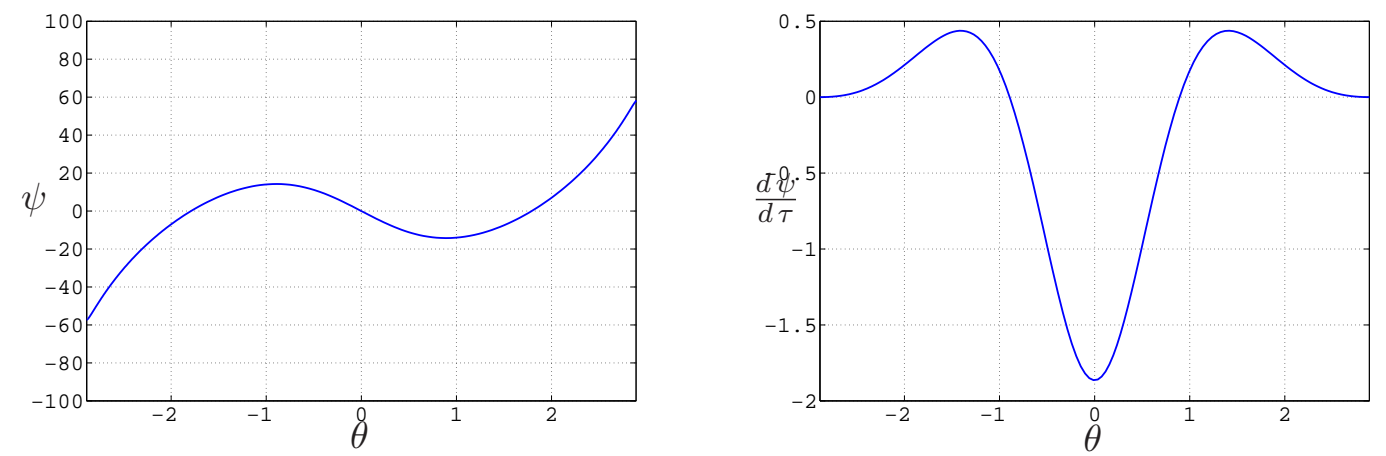

Figure 5. Inertial angle and non dimensional angular velocity during the whole flyby.

The result shows the profile of the inertial angle is antisymmetric respect to $\theta=0$. Furthermore, the value of $\psi$ is close to 0 in the neighborhood of the periapsis (being exactly zero in the periapsis) 
which represents a convenient profile in terms of the generation of electrodynamic force. In turn, the desired final value of the angular velocity has been obtained. Nevertheless, it experiences a large excursion reaching its minimum at the periapsis and being its profile symmetric respect to $\theta=0$.

Other aspects that should be assessed include the sensitivity of the solution which has been obtained. In Figure 6 the evolution of the inertial angle and the non dimensional angular velocity is presented when the initial condition in $\psi$ is modified by $1 \%$ and compared to the nominal solution. From the results of Figure 6, we can conclude that slight initial deviations from the nominal value
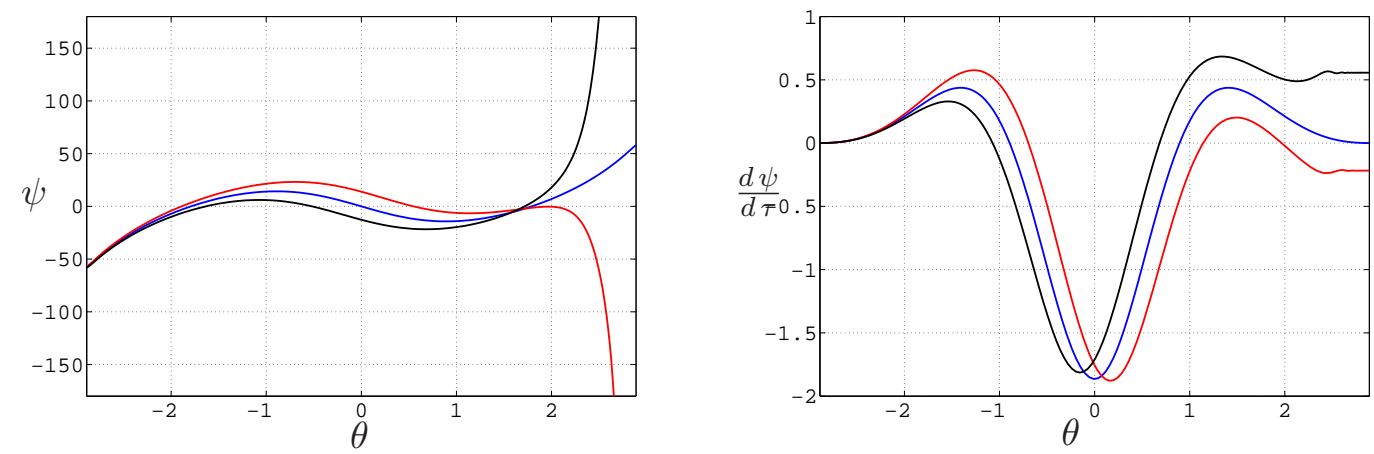

Figure 6 Comparison of the different evolution of the angular variables regarding the initial condition in $\psi$. The nominal solution (blue line) is obtained with $\psi_{0}^{\text {nominal }}=$ $-57.2^{\mathbf{0}}$, while the red line is obtained with $\psi_{0}=1.01 \psi_{0}^{\text {nominal }}$ and the black one $\psi_{0}=$ $0.99 \psi_{0}^{\text {nominal. }}$.

in the initial angle yields an important difference in the final non dimensional angular velocity.

Finally we should explore the situation when electrodynamic forces are considered. A comparison between the nominal solution and the capture trajectory is presented in Figure 7. The initial angle $\psi_{0}$ is the same in both cases. The control variable $\Omega$ has been set to zero during the whole drag arc.
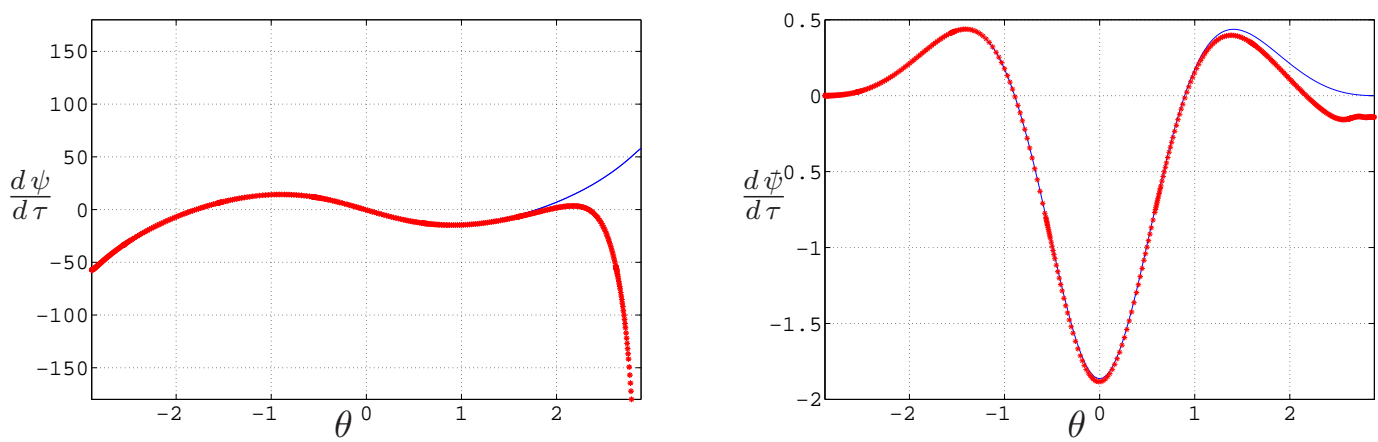

Figure 7 Comparison of the different evolution of the angular variables regarding whether the electrodynamic forces are present (red curve) or not (blue line).

As it can be noted in Figure 7, the effect of the electrodynamic torque during the drag arc has an effect of the same order as the small perturbations in the initial value of the inertial angle $\psi$. 
The electrodynamic torque can be controlled using the free parameter $\Omega_{t}$ as a control variable. In particular, we can cancel the value of the electrodynamic torque adjusting the value of $\Omega_{t}$ to $\Omega_{S B E T}$, the value of the non dimensional load for a self balanced electrodynamic tether (SBET) ${ }^{16,2,1}$. In fact, since the electrodynamic perturbation torque no longer affects the attitude dynamics, this control strategy will lead to an inertial angle profile identical to that showed in Figure 5 when the appropriate initial condition is chosen. Additionally, small variations of $\Omega_{t}$ around $\Omega_{S B E T}$ can be performed to correct the attitude when the actual inertial angle differs from the nominal one.

\section{THERMAL ANALYSIS}

In Gallagher et al., ${ }^{3}$ overheating of the tether turned out to be one of the limiting factors for Jupiter capture. That is the reason why we will carry out a thermal analysis to estimate the effect of the heating problem on the tether.

The energy equation determining the temperature $T(s)$ of an element of tether length $\delta s$ is

$d_{t} h_{t} \delta s \times \rho_{t} c_{t} \frac{\partial T}{\partial t}=\dot{Q}_{t h} \delta s+\dot{Q}_{4} \delta s+\dot{Q}_{x_{x}} \delta s+\dot{Q}_{A} \delta s-2 d_{t} \delta s \times \epsilon_{t} \sigma_{B} T^{4}+d_{t} h_{t} \delta s \times K_{t} \frac{\partial^{2} T}{\partial s^{2}}$

where $d_{t}$ and $h_{t}$ are the width and thickness of the tether tape; $\rho_{t}, c_{t}, \epsilon_{t}$ and $K_{t}$ are the tether density, specific heat, emissivity and thermal conductivity; $\sigma_{B}$ is the Stefan-Boltzmann constant $\left(\sigma_{B}=5.68 \cdot 10^{-8} \mathrm{~W} /\left(\mathrm{m}^{2} \mathrm{~K}^{4}\right)\right)$; and the contributions to the thermal energy are:

- Internal heating power, $\dot{Q}_{t h} \delta s$. It is made of two contributions ${ }^{6}$, one due to the ohmic dissipation and other due to the impact of collected electrons:

$$
\dot{Q}_{t h} \delta s=\frac{I(s)^{2}}{\sigma_{c} d_{t} h_{t}} \delta s+\Phi \frac{d I(s)}{d s} \delta s
$$

where $\sigma_{c}$ is the resistivity of the tether, $I(s)$ is the intensity and $\Phi$ is the bias between plasma and tether. Using the non dimensional variables defined in the study of the intensity along the tether $I(s)=E_{m} \sigma_{c} d_{t} h_{t} i, \Phi=\varphi E_{m} L_{\star}$ and $s=\xi L_{\star}$, the result is:

$$
\dot{Q}_{t h} \delta s=E_{m}^{2} \sigma_{c} d_{t} h_{t}\left[i^{2}+\varphi \frac{d i}{d \xi}\right] \delta s
$$

- Jupiter infrared radiation, $\dot{Q}_{4}$. The form of this contribution is ${ }^{17}$ :

$$
\dot{Q}_{\Psi} \delta s=2 d_{t} \delta s \times F^{o} \sigma_{B} T_{4}^{4} \epsilon_{t}
$$

where $F^{o}$ is the view factor of the tether for Jupiter, and $T_{4}$ is the black body temperature of Jupiter.

- Sun radiation, $\dot{Q}_{\text {s }}$. The expression for the Sun radiation can be written as ${ }^{17}$ :

$$
\dot{Q}_{\text {安 }} \delta s=d_{t} \delta s \times \sigma_{a} \Psi \Phi_{\text {; }}
$$

where $\sigma_{a}$ is the tether absorption coefficient, $\Psi$ is the shining factor and $\Phi_{\text {好 }}$ is the solar flux. 
- Jupiter albedo radiation $\dot{Q}_{A}$. Its intensity is given by ${ }^{17}$ :

$$
\dot{Q}_{A} \delta s=2 d_{t} h_{t} \delta s \times F^{o} \tau_{A} \Phi_{\text {安 }} \sigma_{a} \cos \varsigma
$$

where $\tau_{A}$ denotes the Jupiter albedo and $\varsigma$ represents the Sun-zenith angle, i.e., the reflection angle of Sun rays on Jupiter.

To evaluate the importance of each contribution, we will substitute (57-60) in (55), using the non dimensional variables $\eta=s / L_{t}$ and $\tau=t / \tau_{c}$ where $\tau_{c}=h_{0}^{3} / \mu_{\psi_{4}}^{2}$. The latter is the characteristic time of the hyperbolic initial trajectory. This yields

$$
\begin{aligned}
\frac{\partial T(\eta, \tau)}{\partial \tau}= & \frac{E_{m}^{2} \sigma_{c} \tau_{c}}{\rho_{t} c_{t}}\left[i^{2}(\xi)+\varphi(\xi) \frac{d i(\xi)}{d \xi}\right]-\frac{\epsilon_{t} \sigma_{B} \tau_{c}}{h_{t} \rho_{t} c_{t}}\left[T^{4}(\eta, \tau)-2 F^{o} T_{\psi}^{4}\right]+ \\
& +\frac{\Phi_{h_{t}} \sigma_{a} \tau_{c}}{h_{t} \rho_{t} c_{t}}\left[\Psi+2 F^{o} \tau_{A} \cos \varsigma\right]+\frac{K_{t} \tau_{c}}{L_{t}^{2} \rho_{t} c_{t}} \frac{\partial^{2} T(\eta, \tau)}{\partial \eta^{2}}
\end{aligned}
$$

The relation between both non dimensional lengths is $\xi=\ell_{t} \eta$. Using the values of the aluminum: density, $\rho_{t}=2700 \mathrm{~kg} / \mathrm{m}^{3}$; specific-heat, $c_{t} \approx 900 \mathrm{~J} /(\mathrm{kg} \mathrm{K})$; thermal conductivity, $K_{t} \approx 2.37$ $\mathrm{J} /(\mathrm{m} \mathrm{s} \mathrm{K})$; resistance, $\sigma_{c}=3.7668 \cdot 10^{7} 1 /(\Omega \mathrm{m})$ and considering that $\tau_{c}$ is in the interval $\tau_{c} \in$ $\left[5.4 \cdot 10^{3}, 14 \cdot 10^{3}\right] \mathrm{s}$, the order of magnitude of the conductivity term in $(61)$ is

$$
\frac{K_{t} \tau_{c}}{L_{t}^{2} \rho_{t} c_{t}} \frac{\partial^{2} T(\eta, \tau)}{\partial \eta^{2}} \sim \frac{K_{t} \tau_{c}}{L_{t}^{2} \rho_{t} c_{t}} \sim 10^{-12}
$$

Hence, we don't take this term into account hereafter. We are interested, in first place, in knowing the steady temperature before the capture manoeuvre. In that situation, the left term of the equation vanishes (by assumption the temperature does not vary) and there is no internal heating because the electrodynamic tether is switched off. Therefore, the stationary temperature $T_{0}$ :

$$
T_{0}^{4}=2 F^{o} T_{4}^{4}+\frac{\sigma_{a} \Phi}{\epsilon_{t} \sigma_{B}}\left[\Psi+2 F^{o} \tau_{A} \cos \varsigma\right]
$$

The Jupiter's black body temperature is about $T_{4} \approx 110 \mathrm{~K}{ }^{18}$ and the solar flux at Jupiter is about $\Phi_{\text {保 }} \approx 50.5 \mathrm{~W} / \mathrm{m}^{2}{ }^{18}$. The values of other parameters which are involved are more difficult to specify. We will take approximations for all of them. The view factor $F^{o}$ can vary between 0 and 1 ; we will take $F^{o} \approx 0.5$ since it is a conservative limit for vertical tethers. ${ }^{17}$ Besides, we neglect variations on the Jupiter albedo and we will consider an average value $\tau_{A} \approx 0.34^{18}$. The emissivity and the absorption coefficients of the aluminium are in the ranges: $\epsilon_{t} \in[0.02,0.45]^{17}$ and $\sigma_{a} \in[0.1,0.9]^{17}$. We then choose values $\epsilon_{t} \approx 0.4, \sigma_{a} \approx 0.5$. Finally, we will consider the shining factor $\Psi \approx 0.5$ and the Sun-zenith angle such that $\cos \varsigma \approx 0.5$. In these conditions, the value of the stationary temperature before the capture manoeuvre is: $T_{0} \approx 172 \mathrm{~K}$. Further, we can neglect the variations of all these parameters during capture so that we can rewrite the equation (61) as follows:

$$
\frac{\partial \mathcal{T}(\eta, \tau)}{\partial \tau}=\frac{E_{m}^{2} \sigma_{c} \tau_{c}}{\rho_{t} c_{t} T_{0}}\left[i^{2}(\xi)+\varphi(\xi) \frac{d i(\xi)}{d \xi}\right]-\frac{\epsilon_{t} \sigma_{B} \tau_{c} T_{0}^{3}}{h_{t} \rho_{t} c_{t}}\left[\mathcal{T}^{4}(\eta, \tau)-1\right]
$$

where $\mathcal{T}(\eta, \tau)=T(\eta, \tau) / T_{0}$. Since the conductivity along the tether is negligible, we are assuming that $\mathcal{T}(\eta, \tau) \equiv \mathcal{T}(\tau)$. Then, we can integrate the equation along the tether. Considering that:

$$
\int_{0}^{\ell_{t}}\left(i^{2}(\xi)+\varphi(\xi) \frac{d i}{d \xi}\right) d \xi=\int_{0}^{\ell_{t}} i^{2} d \xi+\varphi i_{0}^{\ell_{t}}-\int_{0}^{\ell_{t}}\left(i^{2}-1\right) d \xi=\varphi_{C} i_{C}+U_{1}
$$


the final form of the energy equation will be:

$$
\frac{d \mathcal{T}(\tau)}{d \tau}=\frac{E_{m}^{2} \sigma_{c} \tau_{c}}{\rho_{t} c_{t} T_{0}} \frac{\left(\varphi_{C} i_{C}+U_{1}\right)}{\ell_{t}}-\frac{\epsilon_{t} \sigma_{B} \tau_{c} T_{0}^{3}}{h_{t} \rho_{t} c_{t}}\left[\mathcal{T}^{4}-1\right]
$$

Because of the definition of the non dimensional temperature $\mathcal{T}$, the initial condition at the beginning of the capture manoeuvre $\mathcal{T}(0)=1$. One of the results which can be obtained from the equation (65) is an estimation of the maximum value of the temperature during the capture. Imposing the condition: $d \mathcal{T} / d \tau=0$ yields to:

$$
\mathcal{T}_{\text {max }}^{4}=1+\frac{\sigma_{c} h_{t}}{\epsilon_{t} \sigma_{B} T_{0}^{4}}\left[E_{m}^{2} \frac{\left(\varphi_{C} i_{C}+U_{1}\right)}{\ell_{t}}\right]_{\max }
$$

In Figure 8 the value of $T_{\max }=\mathcal{T}_{\max } T_{0}$ is shown as a function of the periapsis radius of the initial hyperbolic trajectory, when we suppose the ideal case in the current collection problem: $\varphi_{C}=0$ and we consider the attitude is controlled in such a way that $\phi \equiv 0$. The tether is made of aluminium and its length is $100 \mathrm{~km}$, the total mass of the system being $3075.5 \mathrm{~kg}$.

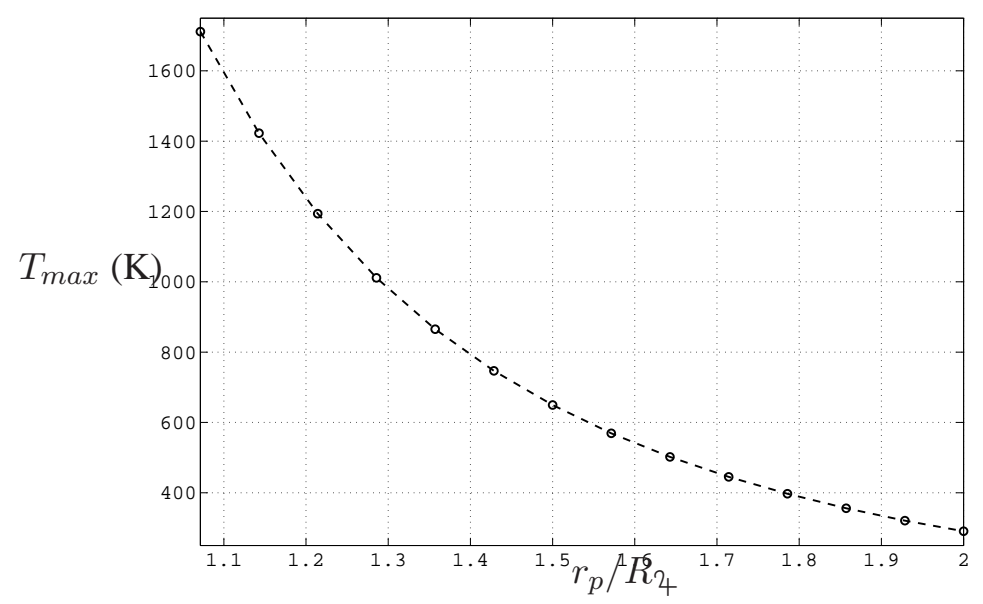

Figure 8. Maximum temperature as a function of the periapsis of the initial hyperbolic trajectory.

Taking into account that the melting temperature or the aluminium is about $930 \mathrm{~K}$, the periapsis of the initial hyperbolic trajectory should be greater than say $1.4 R_{4}$ for this particular tether. The measures we can implement to reduce the maximum temperature as well as the intensity are: increasing the value of $r_{p}$, reduce the value of $L_{t}$ or $h_{t}$. Since the intensity is lower along with the maximum temperature, it is more difficult to perform the capture. Additionally, there are other measures which can be taken affecting only the thermal analysis like: increasing the value of $\epsilon_{t}$, decreasing the value of $\sigma_{a}$ or performing the capture in such a way that the periapsis of the trajectory was shadowed by Jupiter (in such a case $\Psi_{\text {o }}=0$ and $\varsigma=\pi / 2$ ). Even though these changes would have a minor effect.

\section{Operational Limits}

According to what has been said, it is possible to establish the operational limits of a specific tethered system concerning the maximum allowed temperature of the cable material. That constraint 
imposes an upper limit on the length of the tether, once the other parameters of the system are fixed. On the other hand, in order to perform a capture a minimum value of the tether length will be needed. Both restrictions allow to draw the parametric field where the capture can be carried out without reaching too high temperatures.

In Figure 9, the operational limits for a specific non rotating tether are shown. The characteristics of the tether are: the cable is made of aluminium, its width is $3 \mathrm{~cm}$ and its thickness is $0.05 \mathrm{~mm}$. The total mass of the system is the tether mass plus $500 \mathrm{~kg}$. Moreover, a hypothesis has been made concerning the attitude dynamics, assuming the tether follows the nominal attitude defined in Figure 5 during the capture. Regarding the constraints, the maximum allowable temperature has been considered to be $80 \%$ of the melting temperature of the aluminium, and besides the limit of the capture, curves showing the length needed to achieve final elliptical orbits with a period of 100 and 50 days has been represented as well.

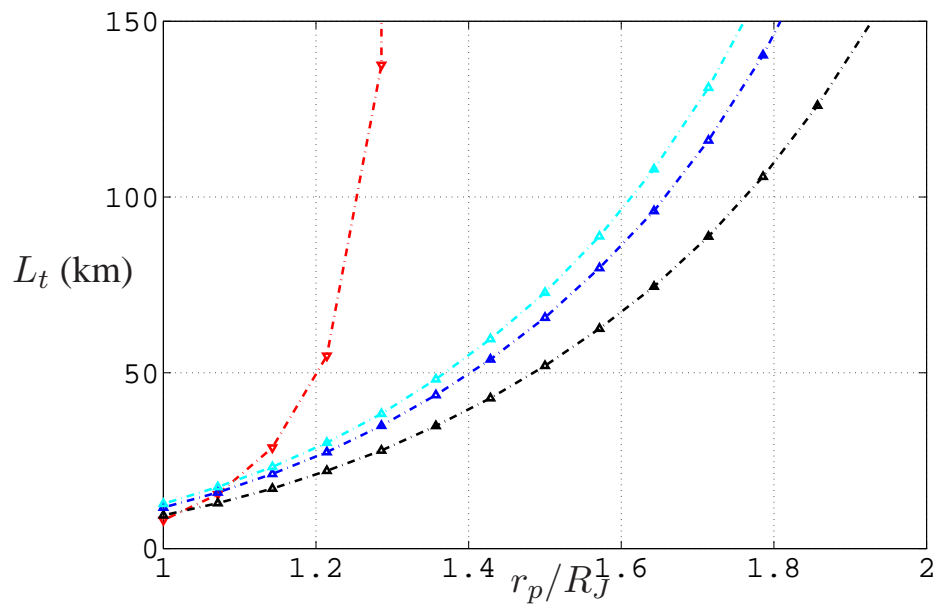

\begin{abstract}
Figure 9 Operational limits of a tethered system performing a capture at Jupiter. The red line is the upper limit due to the maximum temperature the tether can reach (in this case, it has been considered the $80 \%$ of the melting temperature). The black line corresponds to the lower limit needed to perform a capture, while the blue and cyan lines correspond to the length needed to obtain a final elliptical orbit with a period of 100 and 50 days, respectively.
\end{abstract}

As it can be observed, the margin of operation vanishes when the radius of periapsis is close to the radius of Jupiter and it is broader for larger values of that parameter. Nevertheless, as the radius of periapsis increases, the necessary lengths are also larger.

\title{
CONCLUSIONS
}

A derivation of a method to compute with accuracy the effect of the electrodynamic forces in a flyby of Jupiter has been carried out in this paper. This method accounts for both center of mass and attitude motion and it can be applied to other scenarios where low thrust is involved by simply changing the force model, e.g., aerodynamic drag. Nevertheless, it is especially well suited for the case of study in this work due to the great coupling between the attitude and center of mass dynamics.

Using the above-mentioned method, numerical simulations of the behavior of the tethered system 
can be performed easily and precisely. The results have been compared to those found in the literature and the possibility of complete a capture manoeuvre by means of only a electrodynamic tether has been corroborated. Furthermore, a new strategy is proposed to perform the capture. Accordingly, there are two main options to face the design of the tether manoeuvre from the point of view of its attitude profile: on one hand, rapidly rotating tethers with a spin pointing in the direction of the magnetic field at the equator and, on the other hand, a self-balanced non rotating electrodynamic tether with an attitude profile defined in Figure 5. Both procedures present advantages and disadvantages which are summarized in what follows. The rotating tether concept does not need control to keep its operability and the tension required to maintain the cable straight is provided naturally by the centrifugal force. Nevertheless, it presents also drawbacks since the spin should be obtained by means of thrusters and the implementation necessary to change the direction of the current each half revolution is complex. In turn, the non rotating scheme has a better efficiency and less complexity from the point of view of the hardware. However, the control is compulsory and the needed tension to keep the tether tight considering the lateral forces that act on the cable requires further study. Finally, the operational limits for these strategies have been provided considering the thermal constraints and the characteristics of the capture. The results show that it is feasible to perform a capture with tethers of reasonable characteristics in broad areas of the field of parameters (mainly, $v_{\infty}$ and $\left.r_{p}\right)$.

\section{ACKNOWLEDGMENTS}

This work was carried out in the framework of the research project entitled Propagation of orbits, advanced orbital dynamics and use of space tethers (ESP2007-64068) supported by the DGI of the Spanish Ministry of Education and Science.

\section{REFERENCES}

[1] Peláez J. and Scheeres D.J., "On the control of a permanent tethered observatory at Jupiter," 2007 AAS/AIAA Astrodynamics Specialist Conference, Mackinac Island, Michigan, No. Paper AAS07-369, August 19-23 2007.

[2] Peláez and Scheeres D.J., "A permanent tethered observatory at Jupiter. Dynamical analysis," Advances in the Astronautical Sciences, Vol. 127, 2007, pp. 1307-1330.

[3] Gallagher,D.L.; Johnson,L.; Moore,J.; Bagenal,F., "Electrodynamic Tether Propulsion and Power Generation at Jupiter," 7 NASA/TP-1998-208475, NASA.

[4] Sanmartín, J.R.; Lorenzini, E.C., "Exploration of the Outer Planets Using Tethers for Power and Propulsion," Journal of Propulsion and Power, Vol. 21, may-june 2005, pp. 573-576.

[5] van Dijk, A.; Kruijff, M; van der Heide, E.J. \& Lebreton, J.P., "LeBRETON - A lightweight bare rotating tether system for Jovian atmospheric entry," 54th International Astronautical Congress of the International Astronautical Federation (IAF) (A. I. o. Aeronautics and I. Astronautics, eds.), Sep. 29 Oct. 32003.

[6] Sanmartin, J. R.; Charro, M.; Bramanti, C.; Bombardelli, C., "Electrodynamic Tether Microsats at the giant planets," Ariadna Ariadna Study/05/3203, ESA.

[7] J. Sanmartín and R. Estes, "The Orbital-Motion-Limited Regime of Cylindrical Langmuir Probes," Physics of Plasmas, Vol. 6, jan 1999, pp. 395-405.

[8] Sanmartín, J.R. and Martínez-Sánchez, M. and Ahedo, E., "Bare Wire Anodes for Electrodynamic Tethers," Journal of Propulsion and Power, Vol. 9, No. 3, 1993, pp. 353-360.

[9] M. Martínez-Sanchez and J. R. Sanmartín, "Artificial auroral effects from a bare conducting tether," Journal of Geophysical Research, Vol. 102, Dec. 1997, pp. 27257-27264.

[10] Divine, N. and Garret, H.B., "Charged Particle Distributions in Jupiter's Magnetosphere," Journal of Geophysical Research, Vol. 88, No. A9, 1983, pp. 6889-6903.

[11] Khurana, K.K., "Euler Potential Models of Jupiter's Magnetospheric Filed," Journal of Geophysical Research, Vol. 102, 1997, pp. 11295-11306. 
[12] Bagenal, F., "Empirical Model of the Io Plasma Torus: Voyager Measurements," Journal of Geophysical Research, Vol. 99, 1994, pp. 11043-11062.

[13] Peláez J. and Sanjurjo-Rivo M., "Power Generation using Self-Balanced Electrodynamic Tethers in Debris Mitigation Scenarios," Proceedings of the 17 th AAS/AIAA Space Flight Mechanics Meeting, 2007.

[14] D. J. Scheeres, S. J. Ostro, R. A. Werner, E. Asphaug, and R. S. Hudson, "Effects of Gravitational Interactions on Asteroid Spin States," Icarus, Vol. 147, Sept. 2000, pp. 106-118.

[15] D. J. Scheeres, "Changes in Rotational Angular Momentum due to Gravitational Interactions between Two Finite Bodies," Celestial Mechanics and Dynamical Astronomy, Vol. 81, 2001, pp. 39-44.

[16] J. Peláez, "Self balanced electrodynamic tethers," Proceedings of The 2004 AAS/AIAA Astrodynamics Specialist Conference and Exhibit, Providence, Rhode Island, No. Paper AIAA 2004-5309, 2004.

[17] M. Cosmo and E. Lorenzini, "Tethers in Space Handbook," Special Report under Grant NAG8-1160 Third Edition, NASA Marshall Space Flight Center, Dec. 1997.

[18] Jupiter Fact Sheet, "http://nssdc.gsfc.nasa.gov/planetary/factsheet/jupiterfact.html," 